PROCEEDINGS OF THE

AMERICAN MATHEMATICAL SOCIETY

Volume 125, Number 11, November 1997, Pages 3275-3278

S 0002-9939(97)03973-7

\title{
INVARIANT SUBSPACES OF THE MAXIMAL DOMAIN OF THE FOURIER TRANSFORM
}

\author{
GILBERT MURAZ AND PAWEL SZEPTYCKI
}

(Communicated by J. Marshall Ash)

\begin{abstract}
Translation invariant subspaces of the maximal domain of the Fourier transform (the amalgam of $l^{2}$ with $L^{1}$ ) are characterised: it turns out that in this case all measurable subsets of the dual space are sets of spectral synthesis.
\end{abstract}

The problem of characterizing translation invariant subspaces of function spaces on a topological group by means of the Fourier transform goes back to Wiener and has been considered by many authors. For some information about the classical aspects of the subject we refer to $[\mathrm{E}]$ and $[\mathrm{K}]$. Some results in this direction pertaining to $L^{p}$ spaces were recently obtained by A. Olevskii \& al., [O].

The aim of this note is to show that the translation invariant subspaces of the maximal domain of the Fourier transform, the amalgam space $l^{2}\left(L^{1}\right)$, are of the form predicted by Wiener's theorem for the space $L^{2}$. This brings forth yet another aspect of the maximal domain as a natural space in which to study the Fourier transform: the complications with sets of spectral synthesis in some spaces other than $l^{2}\left(L^{1}\right), L^{1}$, for example, do not seem to be present in this case.

We present the result in the case where the underlying measure space is $\mathbf{R}$ with the Lebesgue measure. The argument extends with obvious modifications to $\mathbf{R}^{n}$ and the result remains valid in the setting of locally compact abelian groups (see $[\mathrm{BD}])$. It would also be of interest to study the problem for amalgams $l^{p}\left(L^{q}\right)$ other than $l^{2}\left(L^{1}\right)$ (see the remark at the end of the paper).

We first recall some notations, definitions and known facts concerning the amalgam spaces which will be useful in the proof of our main result. A convenient survey of amalgam spaces can be found in [FS].

Since we only deal with functions on $\mathbf{R}$, we will systematically omit its symbol from our notations, thus a function space $L(\mathbf{R})$ will be denoted by $L$, the integral $\int_{-\infty}^{\infty}$ will be denoted by $\int$ etc.

For $1 \leq p, q<\infty$ the amalgam space $l^{p}\left(L^{q}\right)(\mathbf{R})$ is defined as the subspace of $L_{l o c}^{q}(\mathbf{R})$ of all functions $f$ with finite norm

$$
\left[\sum_{n=-\infty}^{\infty}\|f\|_{L^{q}(n, n+1)}^{p}\right]^{\frac{1}{p}} .
$$

Received by the editors August 29, 1995 and, in revised form, May 20, 1996.

1991 Mathematics Subject Classification. Primary 42A38, 43 A30.

Key words and phrases. Fourier transform, maximal domain.

Supported in part by the General Research Fund, University of Kansas.

(C)1997 American Mathematical Society 
There is an obvious modification of this definition if one (or both) of the parameters $p, q$ is equal to $\infty$.

Of particular interest is the amalgam $l^{2}\left(L^{1}\right)$ which is the maximal solid domain of the Fourier transform in the sense explained in the following proposition.

We denote by $F$ the Fourier transform given by the usual integral operator (with the kernel $\frac{1}{\sqrt{2 \pi}} \exp (-i x y)$ ), by '^' the Fourier transform in $S^{\prime}$ and by $L^{0}$ the space of measurable, finite a.e. scalar functions on $\mathbf{R}$ equipped with the (vector metric) topology of convergence in measure on all subsets of finite measure. Recall that a set $V \subset L^{0}$ is solid if $f \in V$ implies that $[f]:=\left\{g \in L^{0} ;|g| \leq|f|\right.$ a.e. $\} \subset V$. A topological vector subspace (or subgroup) of $L^{0}$ is solid (or locally solid) if its topology is given by a base of neighborhoods of the origin which are solid sets.

Proposition 1. i) $l^{2}\left(L^{1}\right)$ is the maximal solid topological vector subspace of $L^{0}$ to which the (unbounded) operator $F: L^{1} \subset L^{0} \rightarrow L^{0}$ can be extended by continuity.

ii) $f \in l^{2}\left(L^{1}\right)$ if and only if $[f]^{\wedge} \subset L_{l o c}^{1}$.

Remarks. 1. For details concerning i) see [S1] and for ii) see [S2].

2. i) implies, a fact that is readily checked directly, that $L^{1}+L^{2} \subset l^{2}\left(L^{1}\right)$. This inclusion is proper.

3. The space $L_{l o c}^{1}$ in ii) could be replaced by $L^{0}$ : it is implicit that $L^{0} \cap S^{\prime} \subset L_{l o c}^{1}$.

4. It can be shown (see e.g. [S1]) that actually $\widehat{l^{2}\left(L^{1}\right)} \subset l^{\infty}\left(L^{2}\right)$.

5. Similarly to $l^{2}\left(L^{1}\right)$ one can introduce the amalgam space $l^{2}(M)$ of all (Borel) measures $\mu$ on $\mathbf{R}$ such that $\sum|\mu([n, n+1))|^{2}<\infty$, with the obvious norm. It is still true that this is the largest solid space of measures, whose Fourier transforms are functions and that $\widehat{l^{2}(M)} \subset l^{\infty}\left(L^{2}\right)$.

The dual space to $l^{2}\left(L^{1}\right)$ can be identified with the space $l^{2}\left(L^{\infty}\right)$ with the usual pairing $\langle f, g\rangle=\int f(x) g(x) d x, f \in l^{2}\left(L^{1}\right), g \in l^{2}\left(L^{\infty}\right)$. It is obvious that $l^{2}\left(L^{\infty}\right) \subset$ $l^{2}\left(L^{1}\right)$.

It follows in particular that for $f \in l^{2}\left(L^{1}\right), g \in l^{2}\left(L^{\infty}\right)$ the convolution $f * g$ is well defined and belongs to $L^{\infty}$. Also, by Remark $4, \hat{f} \hat{g} \in L_{l o c}^{1} \cap S^{\prime}$. In particular, both expressions $\widehat{f * g}$ and $\sqrt{2 \pi} \hat{f} \hat{g}$ are distributions in $S^{\prime}$. The next proposition asserts (as one would expect) that these two distributions are equal.

Proposition 2. Let $f \in l^{2}\left(L^{1}\right), g \in l^{2}\left(L^{\infty}\right)$ and $\varphi \in C_{0}^{\infty}$. Then

$$
\iint \hat{\varphi}(x-y) f(x) g(y) d x d y=\sqrt{2 \pi} \int \hat{f}(x) \hat{g}(x) \varphi(x) d x .
$$

In other words $\widehat{f * g}=\sqrt{2 \pi} \hat{f} \hat{g}$.

Proof. The identity is true if $f$ and $g$ have compact supports. The general case is obtained by approximation; the passage to the limit can be justified as follows.

If $f_{n} \rightarrow f$ in $l^{2}\left(L^{1}\right)$ or in $l^{2}\left(L^{\infty}\right)$ then, by Remark 4 following Proposition 1, $\hat{f}_{n} \rightarrow \hat{f}$ in $l^{\infty}\left(L^{2}\right)$, in particular in $L_{l o c}^{2}$. This justifies passage to the limit in the right-hand side of the identity in question.

Concerning the left-hand side, we have the following inequality:

$$
\begin{gathered}
\left|\iint \hat{\varphi}(x-y) f(x) g(y) d x d y\right| \leq \sum_{n=-\infty}^{\infty} \sum_{m=-\infty}^{\infty}\left|\int_{n}^{n+1} \int_{m}^{m+1} \hat{\varphi}(x-y) f(x) g(y) d x d y\right| \\
\leq \sum_{n=-\infty}^{\infty} \sum_{m=-\infty}^{\infty} \varphi_{n m}\|f\|_{L^{1}(n, n+1)}\|g\|_{L^{\infty}(m, m+1)} \leq\|\Phi\|\|f\|_{l^{2}\left(L^{1}\right)}\|g\|_{l^{2}\left(L^{\infty}\right)}
\end{gathered}
$$


where $\varphi_{n m}=\max \{|\hat{\varphi}(x-y)| ; x \in[n, n+1], y \in[m, m+1]\}$ and $\|\Phi\|$ is the norm of the operator $\Phi$ defined in $l^{2}(\mathbf{Z})$ by the matrix $\left(\varphi_{n m}\right)$. Since $\hat{\varphi}$ is a function of rapid decrease it is easily checked that $\sum_{m=-\infty}^{\infty} \varphi_{n m} \leq C$ and $\sum_{n=-\infty}^{\infty} \varphi_{n m} \leq C$, where $C$ is a constant depending only on $\varphi$. It follows then from Schur's lemma for integral operators (in the present case on a purely atomic measure space; see e.g. $[\mathrm{G}]$ for a general statement) that $\|\Phi\|<\infty$ and that the bilinear form appearing on the left-hand side of the identity to be proved is a continuous function of the variables $f, g$.

This completes the proof of the proposition.

We will also need the following result from [AS] (Prop. 2.4):

Proposition 3. Let $L$ be a vector subspace of $L^{0}$. Then there exists a maximal and unique, up to sets of measure 0 , measurable set $E \subset \mathbf{R}$ such that all functions in $L$ vanish on $E$. If $L$ is a complete metric space continuously included in $L^{0}$, then there is a function $u \in L$ such that $u(x) \neq 0$ for a.e. $x \in \mathbf{R} \backslash E$.

We recall that a subspace $V$ of $L^{0}$ is translation invariant if for every real $\tau$ and every $f \in V$ we have $f_{\tau} \in V$, where $f_{\tau}(x)=f(x+\tau)$.

For a measurable $E \subset \mathbf{R}$ we denote $I_{E}=\left\{f \in l^{2}\left(L^{1}\right) ; \hat{f}=0\right.$ a.e. in $\left.E\right\}$.

Theorem. i) For every measurable $E \subset \mathbf{R} I_{E}$ is a closed translation invariant subspace of $l^{2}\left(L^{1}\right)$. ii) If $E=\emptyset$, then $I_{E}=l^{2}\left(L^{1}\right)$. iii) If $V$ is a closed translation invariant subspace of $l^{2}\left(L^{1}\right)$, then there exists a measurable set $E \subset \mathbf{R}$ such that $V=I_{E}$.

Remark. The theorem could be restated by saying that every measurable set in $\mathbf{R}$ is a set of spectral synthesis for $l^{2}\left(L^{1}\right)$. Note however that $l^{2}\left(L^{1}\right)$ is not an algebra under convolution (this is an easy consequence of Remark 4 following Proposition 1).

Proof. i) It is clear that $I_{E}$ is translation invariant. $I_{E}$ is closed because of the basic property of $l^{2}\left(L^{1}\right)$ ( Proposition 1, i)): the mapping ${ }^{\wedge}: l^{2}\left(L^{1}\right) \rightarrow L^{0}$ is continuous.

We next prove ii). We consider the image $\hat{V}$ with the norm induced from $l^{2}\left(L^{1}\right)$. This is a Banach subspace of $L^{0}$ and by Proposition 3 there is a maximal measurable set $E \subset \mathbf{R}$ such that $\hat{f}(x)=0$ a.e. on $E$ for all $f \in V$. Also there is a function $f_{0} \in V$ such that $\hat{f}_{0}(x) \neq 0$ for a.e. $x \in \mathbf{R} \backslash E$. Suppose now that $f_{1} \in l^{2}\left(L^{1}\right)$ is in $I_{E}$; we want to show that $f_{1} \in V$. We use the Hahn-Banach theorem: let $\lambda \in l^{2}\left(L^{1}\right)^{*}$ - the dual of $l^{2}\left(L^{1}\right)$-be such that $\lambda(V)=\{0\}$. Then, as remarked above, $\lambda(f)=\int f g$ for some $g \in l^{2}\left(L^{\infty}\right)$ and for all $f \in l^{2}\left(L^{1}\right)$. Since $V$ is translation invariant, $g * f=0$ for all $f \in V$, in particular for $f=f_{0}$. It follows then from Proposition 2 that $\int \hat{f}_{0}(x) \hat{g}(x) \varphi(x) d x=0$ for all $\varphi \in C_{0}^{\infty}$ and therefore $\hat{f}_{0} \hat{g}=0$. Hence $\hat{g}=0$ a.e. outside of $E$ and using again Proposition 2 we conclude that $f_{1} * g=0$. The Hahn-Banach theorem implies that $f_{1} \in V$. This proves ii).

If $E=\emptyset$ then with the notation as above we have $\hat{f}_{0} \neq 0$ a.e. and it follows that $g=0$ a.e. This shows that $V=l^{2}\left(L^{1}\right)$ and proves ii).

Remark. The inclusion $l^{2}\left(L^{p}\right) \subset l^{2}\left(L^{q}\right)$ for $\infty \geq p>q \geq 1$ implies that ${ }^{\wedge}: l^{2}\left(L^{p}\right) \rightarrow$ $l^{\infty}\left(L^{2}\right)$ is continuous and the statement of the theorem remains valid, with the same proof, with $l^{2}\left(L^{1}\right)$ replaced by $l^{2}\left(L^{p}\right), 1<p \leq \infty$. 


\section{REFERENCES}

[AS] N. Aronszajn, P.Szeptycki, On General Integral Transformations, Math. Annalen 163 (1966) 127-154 MR 32:8209

[BD] F. P. Bertrandias, C. Dupuis, Analyse harmonique sur les espaces $l^{p}\left(L^{p^{\prime}}\right)$, Ann. Inst. Fourier XXIX (1979), 189-206.

[E] R.E. Edwards, Fourier Series, vol. II, Holt, Rinehart and Winston, 1967 MR 36:5588

[FS] John J. F. Fournier, J. Stewart, Amalgams of $L^{p}$ and $l^{q}$, Bull A.M.S., 13 (1985) 1-21 MR 86f: 46027

[G] Emilo Gagliardo, On integral transformations with positive kernels, Proceedings AMS, 16, (1965), 429-434

[K] Yitzhak Katznelson, An introduction to Harmonic Analysis, John Wiley and Sons, Inc. 1968 MR 40:1734

[O] A. Olevskii, Translation invariant complemented subspaces in $L^{p}(\mathbb{R})$, Real Analysis Exchange 21 (1995/96), 16-17.

[S1] P. Szeptycki, On functions and measures whose Fourier transforms are functions, Math. Ann. 179 (1968) 31-41 MR 39:710

[S2] P. Szeptycki, On some problems related to the extended domain of Fourier transform, Rocky Mountain J. of Math.,10 (1980) 99-103 MR 82a:42012

Department of Mathematics, Institut Fourier-Grenoble, UFR-UMR 5582, BP 74, 38402 St. Martin d'Heres Cedex, France

E-mail address: muraz@fourier.ujf-grenoble.fr

Department of Mathematics, University of Kansas, Lawrence, Kansas 66045

E-mail address: szeptycki@kuhub.cc.ukans.edu 